\title{
Dynamic Mechanical Properties of Polyoxymethylene. IV
}

\author{
Kyosuke Miki \\ Department of Polymer Science, Faculty of Science, \\ Hokkaido University, Sapporo, Japan.
}

(Received April 4, 1970)

\begin{abstract}
Dynamic mechanical measurements are made with a torsion pendulum apparatus over about three decades of frequency in the $\alpha$ relaxation region of the single crystals of polyoxymethylene. Real and imaginary parts of complex rigidity are measured in each of the three specimens, that is, a mat of single crystals prepared by gradual cooling; a rod of isothermally crystallized single crystals and a heat-treated sample of the single crystal mat. The composite relaxation curves are made by superposing the dispersion curves of real and imaginary parts of complex rigidity on each of the specimens. The composite relaxation curve of loss tangent is also made by superposing the loss tangent curves for the single crystal mat. The activation energies of the $\alpha$ relaxation evaluated from the temperature dependence of the horizontal shift factor are $37 \mathrm{kcal} / \mathrm{mol}\left(T<40^{\circ} \mathrm{C}\right), 25 \mathrm{kcal} / \mathrm{mol}\left(40^{\circ} \mathrm{C}<T<90^{\circ} \mathrm{C}\right)$ and $38 \mathrm{kcal} / \mathrm{mol}\left(T>90^{\circ} \mathrm{C}\right)$ respectively. The magnitude of vertical shift along the logarithmic rigidity axis is independent of temperature above about $100^{\circ} \mathrm{C}$ and $115^{\circ} \mathrm{C}$ for the single crystal mat and the piled rod of the single crystals, but the magnitude varies linearly with increasing temperature above about $50^{\circ} \mathrm{C}$ for the heat-treated sample of the mat.
\end{abstract}

KEY WORDS Polyoxymethylene/Single Crystal/ $\alpha$ Relaxation/Composite Curve/Activation Energy/Vertical Shift Factor/Surface Layer/ Lattice Defect/Lattice Vibration/

Polyoxymethylene is a simple crystalline polymer which has a chemical structure of $-\left(\mathrm{O}-\mathrm{CH}_{2}\right)_{n^{-}}$. The polymer crystallizes with a hexagonal unit cell in which the molecules have a conformation of $9_{5}$ helix in the repeat distance of $17.3 \AA .^{1,2}$

Dynamic mechanical measurements of meltcrystallized polyoxymethylene have already been made by several investigators. ${ }^{3-5}$ Three mechanical relaxation phenomena, usually labeled as $\alpha, \beta$ and $\gamma$ relaxations in the order of descending temperature respectively, have been found in the melt-crystallized samples.

The $\gamma$ relaxation occurs significantly in the melt-crystallized polyoxymethylene. ${ }^{3}$ It has also been observed in dielectric measurements. ${ }^{6}$ Another relaxation peak, the $\gamma_{2}$ peak, has been observed in the lower temperature region of the $\gamma$ relaxation. $^{7}$ The composite relaxation curves have been formed by superposing the dispersion curves in the $\gamma$ relaxation region. ${ }^{8}$ The timetemperature superposition principle ${ }^{9}$ was applicable to the relaxation. The temperature dependence of the shift factor $a_{T}$ was represented by Williams-Landel-Ferry's equation. ${ }^{10}$

The $\beta$ relaxation of melt-crystallized polyoxymethylene has a weak peak in comparison with the $\alpha$ and $\gamma$ relaxations. ${ }^{3}$ A detailed study has shown that the magnitude of the $\beta$ peak depends on the amount of the absorbed water and the thermal history of the sample. ${ }^{3}$

Folded conformation of polyethylene molecules in the single crystal precipitated from the dilute solution was first proposed by Keller. ${ }^{11}$ Single crystals of polyoxymethylene, precipitated from the dilute solution, were shown by Geil, et al., to consist of hexagonal lamella approximately $100 \AA$ thick. $^{12}$ Dynamic mechanical study of the single crystal mats of polyoxymethylene was first made by Takayanagi and his co-workers. ${ }^{13}$ The $\alpha$ relaxation was found to be more pronounced in the single crystal mats. Single crystals of polyoxymethylene have a relatively simple morphological structure. It is desirable for this reason to examine further the mechanical relaxation of the single crystals in order to clarify the origin of the $\alpha$ relaxation. 


\section{Dynamic Mechanical Properties of Polyoxymethylene}

Dynamic mechanical measurements of meltcrystallized polyoxymethylene have been made over about two decades of frequency using a forced-oscillation torsion pendulum in the $\alpha$ relaxation region. ${ }^{14}$ The composite relaxation curves have been made by superposing the relaxation curves.

This paper is concerned with the dynamic mechanical measurements made over about three decades of frequency in the $\alpha$ relaxation region of the single crystals of polyoxymethylene.

The discussion that follows is based partly upon the experimental results obtained recently in the X-ray diffraction study of polyoxymethylene. A hypothesis concerning the origin of the $\alpha$ relaxation will be proposed. This hypothesis is supported by the experimental facts so far obtained.

The dispersion curves in the $\alpha$ relaxation region of melt-crystallized polyoxymethylene were superposed over a wide range of frequency by performing the vertical and horizontal shifts along the logarithmic rigidity and frequency axes respectively. ${ }^{14}$ The vertical shifts were necessary for the melt-crystallized sample in temperature above approximately $40^{\circ} \mathrm{C}$. The temperature variation of the magnitude of the vertical shift has shown a remarkable anisotropy in the $\alpha$ relaxation region of the drawn and oriented polyoxymethylene..$^{15}$ One of the purposes of this work is to examine whether the vertical shifts are necessary for the superposition of the dispersion curves of the single crystals of polyoxymethylene. This paper is therefore concerned also with the origin of the vertical shift along the logarithmic rigidity axis.

\section{EXPERIMENTAL}

\section{Sample}

A sample of polyoxymethylene, du Pont Delrin $150 \mathrm{X}$, was used in this study.

Single crystals were prepared from a $0.1 \mathrm{wt} \%$ cyclohexanol solution of the sample using ambient cooling. The single crystals were separated from the solvent by gradual cooling. The suspension of the crystals was slowly filtered through a $6 \mathrm{~cm}$-diameter glass filter. When all the crystals were transfered to this filter funnel and the height of the suspension in the funnel reduced to almost $1 \mathrm{~cm}$, a $5.8 \mathrm{~cm}$-diameter Teflon disk was carefully placed on the top of the crystals. A $2.3 \mathrm{~kg}$ weight was placed on the disk and vacuum pulled on the filter flask for about one week. The single crystals were further dried by evacuation in a desiccator for one week at room temperature. Then a small compressive load was applied to the crystals placed between two sheets of Teflon disk using a laminating press at room temperature. The single crystals were finally compressed to a mat of approximately $0.1 \mathrm{~cm}$ thick and $5 \mathrm{~cm}$ in diameter. The density measured with a density gradient tube of xylene$\mathrm{CCl}_{4}$ system was $1.465 \mathrm{~g} / \mathrm{cc}$ at $25^{\circ} \mathrm{C}$. The long period evaluated from the X-ray small angle scattering pattern was approximately $120 \AA$. The $X$-ray small angle scattering pattern indicated that voids of various sizes are included in the mat. This sample was strong enough to be used for mechanical measurements, if handled carefully. The specimens were cut out from the mat using a heated razor.

Another sample was a rod of the isothermally crystallized single crystals. The single crystals were isothermally separated from a $0.05 \mathrm{wt} \%$ cyclohexanol solution of polyoxymethylene at a temperature of $120^{\circ} \mathrm{C}$. The suspension of the crystals was slowly filtered through the glass filter. Repeating the same procedure as above, a single crystal mat of $0.5 \mathrm{~cm}$ thick was obtained. The crystal mat was dried for one week at room temperature in the evacuated desiccator. Then various columns of about $0.8 \mathrm{~cm}$-diameter and $0.5 \mathrm{~cm}$ thickness were gouged out from the mat using a thin brass tube of $0.8 \mathrm{~cm}$ inside diameter and about $20 \mathrm{~cm}$ long. These columns were piled up one after another in the brass tube and a brass rod of $0.75 \mathrm{~cm}$-diameter and about $30 \mathrm{~cm}$ long was inserted into the tube. Then a small compressive load was applied to the pile of crystals with a $2 \mathrm{~kg}$ weight set at the top of the brass rod. A circular rod $1 \mathrm{~cm}$ in length and $0.8 \mathrm{~cm}$ in diameter was finally obtained. A rodlike specimen of $0.1 \mathrm{~cm}$-diameter was cut out from the circular rod for the mechanical measurements.

A specimen cut out from the single crystal mat was annealed at a temperature of $190^{\circ} \mathrm{C}$. A thin rod of the original single crystal mat 
was set in a copper frame $0.1 \mathrm{~cm}$ thick and placed between two sheets of brass plate heated beforehand to approximately $190^{\circ} \mathrm{C}$. Then the brass plates were set in the laminating press controlled at a temperature of $190^{\circ} \mathrm{C}$. A small compressive load was applied to prevent the specimen from curling. The specimen was heated for two minutes in this press. Then it was rapidly cooled to room temperature by setting the plates into a cooling press in which water was circulated. This specimen is hereinafter referred to as the heat-treated single crystal mat.

\section{Mechanical Measurements}

Dynamic mechanical measurements were made by using a forced-oscillation torsion pendulum in an ultra-low frequency range below $1 \mathrm{~Hz}$. Details of the apparatus were described in a previous paper. ${ }^{14}$

The specimens were in the form of a thin rectangular rod, $2.5 \mathrm{~cm}$ long between clamps, 0.3 $\mathrm{cm}$ wide and $0.1 \mathrm{~cm}$ thick.

Maximum shear strain imposed during the measurements was less than $1 \%$. The real and imaginary parts of the complex rigidity were calculated from the phase angle of the oscillation between the driving coil and the pendulum, $\varphi$, and the ratio of the amplitude of the oscillation of the pendulum to that of the driving coil, $p$, using the following equations

and

$$
G^{\prime}(\omega)=q K_{0}\left\{\left(\frac{\cos \varphi}{p}-1\right)+I \omega^{2}\right\}
$$

$$
G^{\prime \prime}(\omega)=q K_{0}\left(\frac{\sin \varphi}{p}\right)
$$

where $q$ is the form factor of the sample, $K_{0}$ the torsional constant of a wire connecting the driving coil and the pendulum, $I$ the moment of inertia of the pendulum and $\omega$ the angular frequency.

The error in the real part $G^{\prime}$ of the complex rigidity was estimated to be $3 \%$ and in the imaginary part $G^{\prime \prime}$ less than $10 \%$.

Temperature of the specimen was controlled by an electric heater set in the sample cell. Temperature was measured with a thermister within $\pm 0.5^{\circ} \mathrm{C}$.

\section{RESULTS}

Figure 1 shows the frequency dependence of the real and imaginary parts of complex rigidity in the $\alpha$ relaxation region for the single crystal

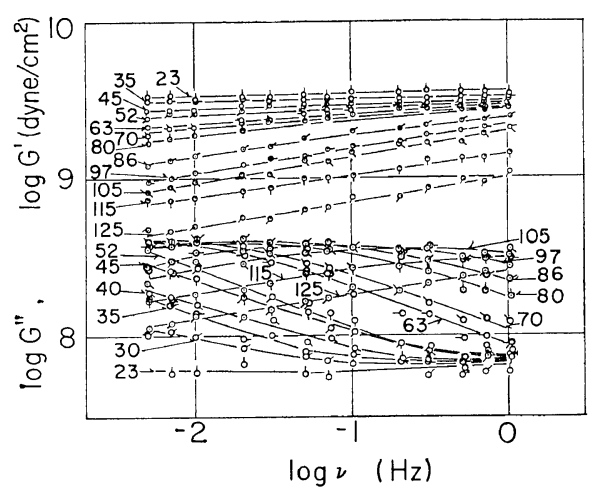

Figure 1. Frequency dependence of real and imaginary parts of complex rigidity, $G^{\prime}$ and $G^{\prime \prime}$. Over a frequency range from $1.0 \mathrm{~Hz}$ to $0.005 \mathrm{~Hz}$ at various temperatures for the single crystal mat of polyoxymethylene prepared by gradual cooling. Temperature is shown for each curve.

mat of polyoxymethylene prepared by gradual cooling. Measurements were made over a frequency range from $1.0 \mathrm{~Hz}$ to $0.005 \mathrm{~Hz}$ at various temperatures between $23^{\circ} \mathrm{C}$ and $125^{\circ} \mathrm{C}$.

Figure 2 shows the frequency dependence of the loss tangent in the same region.

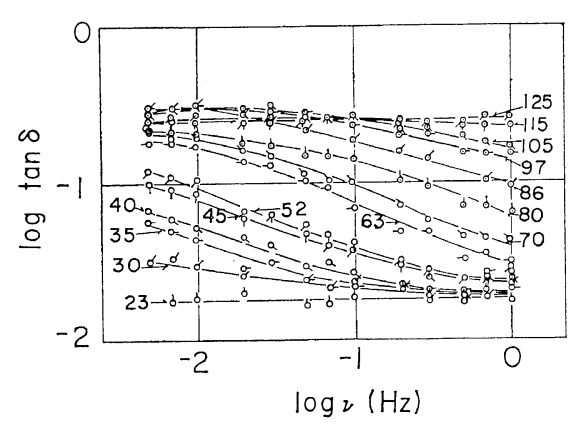

Figure 2. Frequency dependence of loss tangent, $\tan \delta$. Over a frequency range from $1.0 \mathrm{~Hz}$ to $0.005 \mathrm{~Hz}$ at various temperatures for the single crystal mat of polyoxymethylene prepared by gradual cooling. Temperature is shown for each curve.

Figure 3 shows the frequency dependence of the real and imaginary parts of complex rigidity 
in the $\alpha$ relaxation region for the piled rod of the isothermally crystallized single crystals. Measurements were made over a frequency range from $1.0 \mathrm{~Hz}$ to $0.002 \mathrm{~Hz}$ at temperatures between $22^{\circ} \mathrm{C}$ and $139^{\circ} \mathrm{C}$. The measured values of $G^{\prime}$ and $G^{\prime \prime}$ are considerably smaller than those of the single crystal mat. This is due to the numerous voids contained within the piled rod.

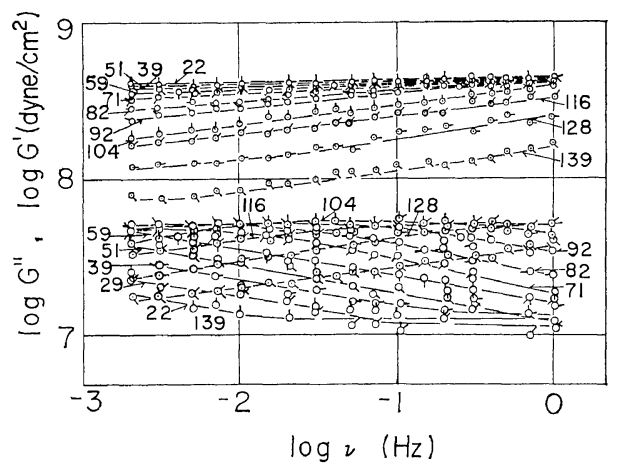

Figure 3. Frequency dependence of real and imaginary parts of complex rigidity, $G^{\prime}$ and $G^{\prime \prime}$. Over a frequency range from $1.0 \mathrm{~Hz}$ to $0.002 \mathrm{~Hz}$ at various temperatures for the piled rod of the isothermally crystallized single crystals. Temperature is shown for each curve.

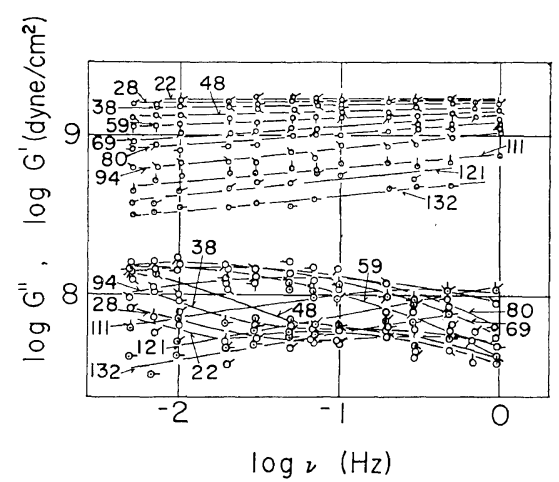

Figure 4. Frequency dependence of real and imaginary parts of complex rigidity, $G^{\prime}$ and $G^{\prime \prime}$. Over a frequency range from $1.0 \mathrm{~Hz}$ to $0.005 \mathrm{~Hz}$ at various temperatures for the heat-treated single crystal mat. Temperature is shown for each curve.

Figure 4 shows the frequency dependence of real and imaginary parts of complex rigidity in the $\alpha$ relaxation region for the heat-treated single crystal mat. Measurements were made over a frequency range from $1.0 \mathrm{~Hz}$ to $0.005 \mathrm{~Hz}$ at temperatures between $22^{\circ} \mathrm{C}$ and $132^{\circ} \mathrm{C}$.

\section{DISCUSSION}

\section{Reduced Variable Method}

Superpositions of the dispersion curves can be performed by applying the method of reduced variables extended to the crystalline polymers, such as melt-crystallized polyoxymethylene. ${ }^{14}$ The real $G^{\prime}$ and imaginary $G^{\prime \prime}$ parts of the complex rigidity in the $\alpha$ relaxation region are reduced to a reference temperature $T_{0}$ by the following equations

$$
\text { and } \begin{aligned}
G^{\prime}(\nu) & =G_{0}{ }^{\prime}\left(\nu a_{T}\right) \exp \left\{-\beta\left(T-T_{0}\right)\right\} \\
G^{\prime \prime}(\nu) & =G_{0}{ }^{\prime \prime}\left(\nu a_{T}\right) \exp \left\{-\beta\left(T-T_{0}\right)\right\}
\end{aligned}
$$

where $G^{\prime}$ and $G^{\prime \prime}$ are the quantities at a temperature $T$ and $G_{0}{ }^{\prime}$ and $G_{0}{ }^{\prime \prime}$ are those at a reference temperature $T_{0}$ respectively. The $\beta$ is a coefficient indicating the temperature dependence of the rigidity and $a_{T}$ is the horizontal shift factor along the logarithmic frequency axis. Since the loss tangent is defined by the formula, $\tan \delta(\nu)=G^{\prime \prime}(\nu) / G^{\prime}(\nu)$, we have the following equation from the eq 3 and 4

$$
\tan \delta(\nu)=\tan \delta_{0}\left(\nu a_{T}\right)
$$

where $\tan \delta$ and $\tan \delta_{0}$ are the quantities at $T$ and $T_{0}$ respectively. This equation demands that the $\tan \delta$ curves superpose by performing only horizontal shifts along logarithmic frequency axis.

Figure 5 shows the composite relaxation curves of real and imaginary parts of complex rigidity for the single crystal mat. The composite curves were made by superposing the $G^{\prime}$ and $G^{\prime \prime}$ curves in Figure 1.

Figure 6 shows the composite curve of $\tan \delta$ made by superposing the $\tan \delta$ curves in Figure 2 . The $\tan \delta$ curves fairly superposed by performing only horizontal shifts along the logarithmic frequency axis. The reference temperature was selected at $70^{\circ} \mathrm{C}$.

Figure 7 shows the composite relaxation curves of $G^{\prime}$ and $G^{\prime \prime}$ for the piled rod of the isothermally crystallized single crystals made by superposing the $G^{\prime}$ and $G^{\prime \prime}$ curves in Figure 3. The reference temperature was selected at $92^{\circ} \mathrm{C}$. 
K. MIKI

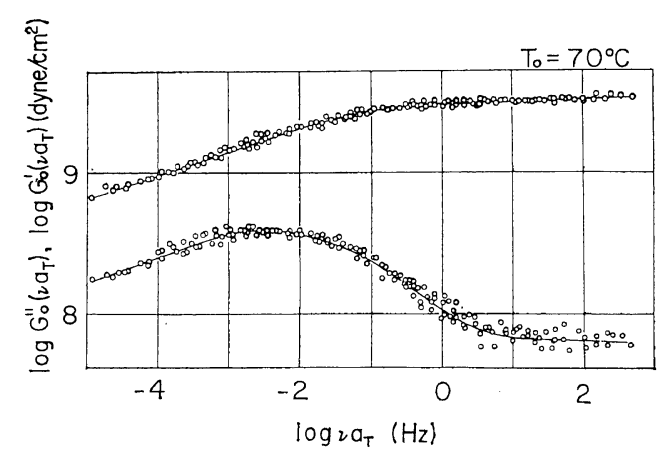

Figure 5. Composite relaxation curves of real and imaginary parts of complex rigidity. Reduced to a reference temperature of $70^{\circ} \mathrm{C}$ for the single crystal mat prepared by gradual cooling.

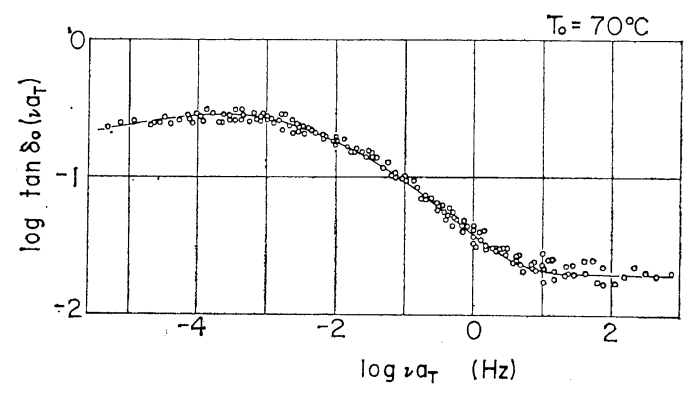

Figure 6. Composite relaxation curve of loss tangent. Reduced to a reference temperature of $70^{\circ} \mathrm{C}$ for the single crystal mat prepared by gradual cooling.

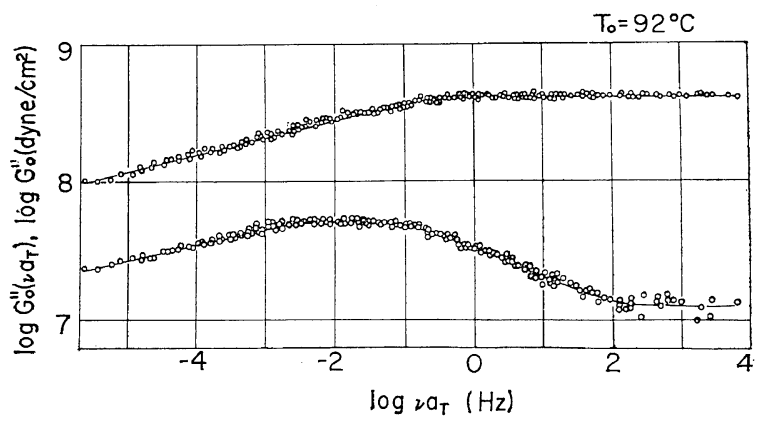

Figure 7. Composite relaxation curves of real and imaginary parts of complex rigidity. Reduced to a reference temperature of $92^{\circ} \mathrm{C}$ for the piled rod of the isothermally crystallized single crystals.

Figure 8 shows the composite relaxation curves of $G^{\prime}$ and $G^{\prime \prime}$ for the heat-treated single crystal

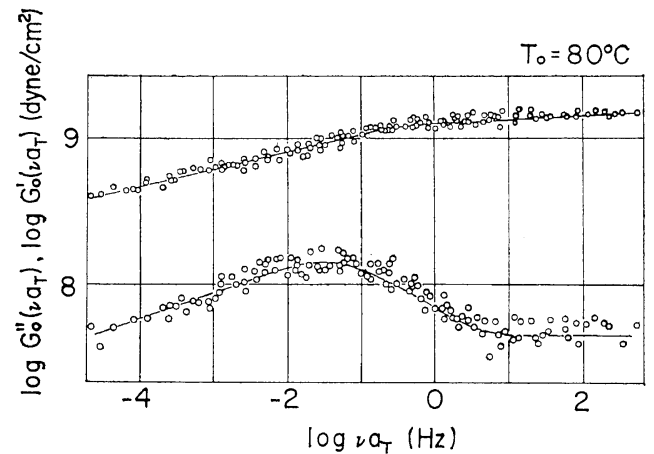

Figure 8. Composite relaxation curves of real and imaginary parts of complex rigidity. Reduced to a reference temperature of $80^{\circ} \mathrm{C}$ for the heat-treated single crystal mat.

mat made by superposing the $G^{\prime}$ and $G^{\prime \prime}$ curves in Figure 4. The reference temperature was selected at $80^{\circ} \mathrm{C}$.

In Figure 9 logarithms of the shift factor $a_{T}$, which represents the magnitude of the horizontal shift along the logarithmic frequency axis, were plotted against the reciprocal of the absolute temperature for each of the specimens. The $a_{T}$ curves of these specimens were denoted by the

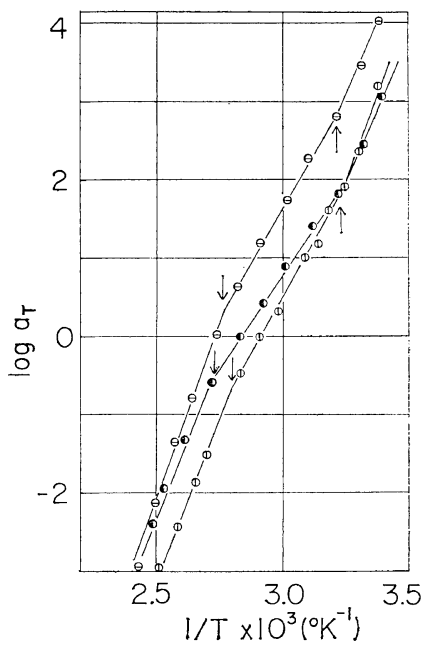

Figure 9. Logarithms of the horizontal shift factor $v s$. reciprocal absolute temperature for each of the specimens. The curves are denoted by the following symbols: (1), the single crystal mat prepared by gradual cooling; $\Theta$, the piled rod of the isothermally crystallized single crystals and 1 , the heat-treated single crystal mat. 


\section{Dynamic Mechanical Properties of Polyoxymethylene}

following symbols: (D) the single crystal mat; $(\ominus)$ the piled rod of the isothermally crystallized single crystals and $(O)$ the heat-treated single crystal mat. The temperature dependence of the relaxation time of the $\alpha$ relaxation was almost identical in spite of the difference in the preparation methods of the specimens. The curves were represented to a close approximation by three straight lines, intersecting at about $90^{\circ} \mathrm{C}$ and $40^{\circ} \mathrm{C}$. The arrows show the intersections of the straight lines. The activation energies were evaluated at approximately $37 \mathrm{kcal} / \mathrm{mol}(T<$ $\left.40^{\circ} \mathrm{C}\right), 25 \mathrm{kcal} / \mathrm{mol}\left(40^{\circ} \mathrm{C}<T<90^{\circ} \mathrm{C}\right)$ and $38 \mathrm{kcal} /$ $\mathrm{mol}\left(T>90^{\circ} \mathrm{C}\right)$ respectively. These values almost coincide with those evaluated for the meltcrystallized and oriented samples of polyoxymethylene. ${ }^{14,15}$

Figure 10 shows the temperature dependence of the magnitude of the vertical shift along the

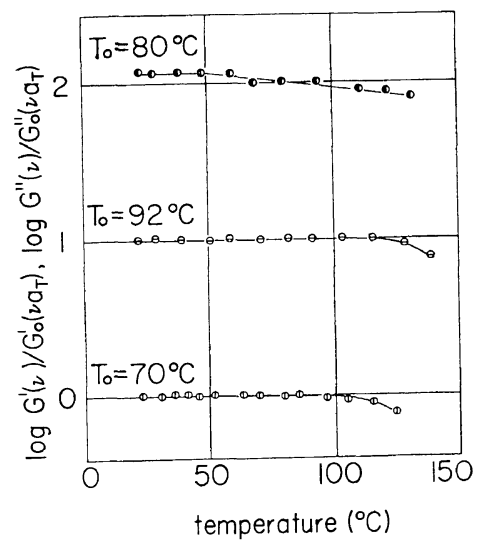

Figure 10. Temperature dependence of the magnitude of the vertical shift along logarithmic rigidity axis for each of the specimens. The curves are denoted by the following symbols: (1), the single crystal mat prepared by gradual cooling; $\ominus$, the piled rod of the isothermally crystallized single crystals and $\boldsymbol{D}$, the heat-treated single crystal mat. The curves have been displaced vertically one decade above that of the preceding one. The reference temperature is shown for each curve.

logarithmic rigidity axis for each of the specimens. The curves have been displaced vertically one decade above that of the preceding one. The symbols have the same meaning as in Figure
9. The magnitudes for $G^{\prime}, \log \left\{G^{\prime}(\nu) / G_{0}{ }^{\prime}\left(\nu a_{T}\right)\right\}$, were almost in agreement with those for $G^{\prime \prime}$, $\log \left\{G^{\prime \prime}(\nu) / G_{0}{ }^{\prime \prime}\left(\nu a_{T}\right)\right\}$, on each of the specimens.

The magnitude of the vertical shift is almost independent of temperature below about $100^{\circ} \mathrm{C}$ and it varies with increasing temperature above $100^{\circ} \mathrm{C}$ for the single crystal mat prepared by gradual cooling. Similarly the magnitude is independent of temperature below about $115^{\circ} \mathrm{C}$ for the piled rod of the isothermally crystallized single crystals. The coefficient $\beta$ in the eq 3 and 4 was equally evaluated to be zero in temperature below $100^{\circ} \mathrm{C}$ and $115^{\circ} \mathrm{C}$ for these specimens. This is irrespective of the difference in the preparation method of the single crystals.

For the heat-treated single crystal mat the magnitude of the vertical shift varies linearly with increasing temperature above about $50^{\circ} \mathrm{C}$. The coefficient $\beta$ in eq 3 and 4 was evaluated to be $0.0039 \mathrm{deg}^{-1}$ from the slope of the curve. This value is considerably smaller than that for the melt-crystallized polyoxymethylene, 0.0094 $\operatorname{deg}^{-1} \cdot{ }^{14}$

\section{$\alpha$ Relaxation}

Takayanagi, et al., found that the position of the maximum of the $\alpha$ relaxation of linear polyethylene is approximately coincident with the temperature where the thermal expansion coefficient of the $a$-axis of the crystal increases. ${ }^{16}$ They proposed on this basis a hypothesis that the $\alpha$ relaxation occurs from the friction among the (2.0.0) planes which takes place under the mechanical deformation of the crystallites.

Wada, et al., measured the dynamic mechanical properties of single crystals of polyethylene using a suspension method. ${ }^{17,18}$ The $\alpha$ relaxation was observed even in the alcohol suspension of the single crystals. They proposed on this basis a hypothesis that the $\alpha$ relaxation comes from the incoherent rotational lattice vibrations of the chain molecules around the $c$-axis of the crystal. Okano $^{19}$ developed a quantitative theory of the crystalline relaxation on this hypothesis by applying Peterlin-Fischer's theory of the thermodynamic stability of the polymer crystals. ${ }^{2021}$ Okano's theory was quantitatively verified by Tsuge. $^{22}$ The intensity of the mechanical or dielectric $\alpha$ relaxation was found to increase with increasing chain length for $n$-paraffin crystals. 
Similar results were found in the relationship between the intensity of the dielectric $\alpha$ relaxation and the thickness of lamella for melt-crystallized polyoxymethylene. ${ }^{23}$

The intensity of the mechanical $\alpha$ relaxation of melt-crystallized polyoxymethylene also increases with increasing thickness of lamella. ${ }^{24}$ This relationship is in qualitative agreement with the requirements of Okano's theory. However the relaxation intensity was found to increase by gamma-ray irradiation on the melt-crystallized polyoxymethylene without increasing the lamella thickness.

Sinnott examined in detail the effect of annealing treatment on the dynamic mechanical properties of single crystal mats of linear polyethylene. ${ }^{25}$ The intensity of the $\alpha$ relaxation was found to decrease with increasing thickness of lamella with such annealing treatment. He concluded on this basis that the $\alpha$ relaxation is associated with the reorientation of the folds from one equilibrium position to another at the surfaces of the lamella in the single crystals of polyethylene.

Takayanagi, et al., found that the intensity of the $\alpha$ relaxation increases with increasing thickness of lamella in the polyethylene single crystals prepared by isothermal crystallization at various temperatures, but that on the other hand it decreases with the increasing thickness of lamella of the single crystals under the annealing treatment. ${ }^{26}$

As described above, many different views have been proposed concerning the origin of the $\alpha$ relaxation of highly crystalline polymers, such as polyethylene and polyoxymethylene.

As shown in Figure 9, the temperature dependence of the relaxation time of the $\alpha$ relaxation for single crystals almost coincides with that for the melt-crystallized polyoxymethylene. ${ }^{14}$ The abrupt change of the activation energy at about $40^{\circ} \mathrm{C}$ has been already found for the meltcrystallized sample. This change occurs even for the single crystals at the same temperature. If the $\alpha$ relaxation is related to the motion of the chain molecules in the crystal, some noticeable change should occur in the thermal motion of the chain molecules of polyoxymethylene in the same temperature region.
Recently the temperature dependence of the $\mathrm{X}$-ray scattering intensity of the Bragg reflection of the (2.1.8) and (1.1.13) planes was measured over a wide range of temperature between $-130^{\circ} \mathrm{C}$ and $150^{\circ} \mathrm{C}$ for the crystals of polyoxymethylene. ${ }^{27}$ The diffraction intensity decreases with increasing temperature and the temperature factor of the intensity in particular increases at a temperature of approximately $40^{\circ} \mathrm{C}$. The thermal expansion coefficient of the spacing of the (1.1.0) plane also increases in the same temperature region.

The temperature dependence of the X-ray small angle scattering intensity was measured over a wide range of temperature between $-100^{\circ} \mathrm{C}$ and $130^{\circ} \mathrm{C}$ for the melt-crystallized polyoxymethylene. $^{28}$ The scattering intensity was found to increase with increasing temperature. The curve of the square root of the intensity plotted against temperature was represented to a close approximation by three straight lines, intersecting at approximately $-60^{\circ} \mathrm{C}$ and $40^{\circ} \mathrm{C}$ respectively. The increase of the scattering intensity was quantitatively interpreted by the temperature variation of the density difference between the crystalline region and the amorphous region up to a temperature of about $40^{\circ} \mathrm{C}$. The temperature of $-60^{\circ} \mathrm{C}$ is coincident with the glass transition temperature of the amorphous region which lies between lamellae. ${ }^{8}$

The further increase of the small angle scattering intensity starting at about $40^{\circ} \mathrm{C}$ could not be ascribed to the temperature variation of the density difference of these two regions only. The increase of the scattering intensity at temperatures above $40^{\circ} \mathrm{C}$ seems to be explained as the increase of the amorphous region caused by the partial melting of the surface layer of the crystalline lamella. ${ }^{29,30}$ However the decrease of the degree of crystallinity has not been observed in polyoxymethylene up to a temperature of about $100^{\circ} \mathrm{C}^{31}$ The further increase of the intensity starting at about $40^{\circ} \mathrm{C}$ should be ascribed to some other origin.

At temperatures above about $40^{\circ} \mathrm{C}$ large amplitude lattice vibrations of the chain molecules occur in the crystal and the thermal expansion coefficient of the crystal lattice significantly increases. $^{27}$ If the amplitude of the thermal vi- 
bration of the chain molecules becomes larger in the surface layer of the lamella than in the inner layer of the lamella, a structural looseness should be caused, especially in the surface layer, with increasing temperature. This structural looseness is closely related to the occurrence of the thermal disorder of the crystal arising from the large amplitude lattice vibration in the surface layer. As the looseness develops in the surface layer, the density decreases in the neighborhood of the fold surfaces of lamella. The further increase in the small angle scattering intensity starting at about $40^{\circ} \mathrm{C}$ might be caused by the decrease of the density due to the large amplitude lattice vibration in the surface layer.

The noticeable change of the activation energy of the $\alpha$ relaxation starting at about $40^{\circ} \mathrm{C}$ should be also connected with the occurrence of the structural looseness of the surface layer of the lamella. The $\alpha$ relaxation of polyoxymethylene is ascribed to the viscoelastic properties of the lamellar crystal arising from the large amplitude lattice vibrations in the surface layer.

The $\alpha$ relaxation at first occurs in the surface layer and subsequently in the inner layer of the lamellar crystal. The relaxation time is shorter in the surface layer than in the inner layer. The long relaxation time range of the relaxation spectrum, which is actually observed at higher temperatures, can be therefore attributed to the inner layer. As shown in Figure 9, the activation energy of the $\alpha$ relaxation of polyoxymethylene increases again at higher temperatures above about $90^{\circ} \mathrm{C}$. This large activation energy may be related to the more ordered structure of the inner layer of the lamellae.

The volume fraction of the surface layer of the crystalline lamella generally decreases with increasing thickness of lamella. If the hypothesis on the origin of the $\alpha$ relaxation is valid, the intensity of the $\alpha$ relaxation should decrease with increasing thickness of lamella. In the radiationinduced solid state polymerized polyoxymethylene, ${ }^{32,33}$ such as poly-trioxane and poly-tetraoxane, the crystals are known to be composed of highly oriented and extended molecules. The thickness of the crystal is very large as compared with that of the single crystal mat or the melt-crystallized sample. The $\alpha$ relaxation has remarkably decreased in intensity in each of the solid-state polymerized samples. ${ }^{34,35}$ This fact supports the above hypothesis.

Complex tensile modulus of the drawn and oriented samples of polyoxymethylene was measured in sheet form as a function of direction. ${ }^{36}$ The imaginary part of complex tensile modulus in the $\alpha$ relaxation region was found to have higher values along the stretched direction than along the normal direction to the stretched axis. The X-ray small angle scattering pattern of the drawn polyoxymethylene is known to indicate a two-point diagram along the meridional direction. ${ }^{15,36}$ This means that the fold surfaces of the lamella are oriented along the normal direction to the stretched axis. ${ }^{37}$ The three layers, that is, the inner and surface layers of the lamella and the amorphous layer, are arranged mainly in series along the stretched direction. If the hypothesis concerning the origin of the $\alpha$ relaxation is justified, the anisotropy of the intensity of the relaxation can be easily explained using this mechanical model.

\section{Temperature Dependence of Rigidity}

Figure 10 shows the temperature dependence of the magnitude of the vertical shift along the logarithmic rigidity axis for the three specimens. This figure represents the temperature variation of the rigidity of the crystals, which is caused independently of the $\alpha$ relaxation.

The temperature variation of the real and imaginary parts of complex rigidity which starts at about $40^{\circ} \mathrm{C}$ for the melt-crystallized polyoxymethylene was theoretically interpreted in accordance with the concept of the "smearing-out effect" of the elastic modulus due to the incoherent lattice vibrations in polymer crystals. ${ }^{14}$ A remarkable anisotropy was found for the temperature dependence of rigidity of the drawn and oriented polyoxymethylene. ${ }^{15}$ The anisotropy was interpreted on the concept of the "smearingout effect" of a component $c_{66}$ of the elastic modulus tensor of the crystal due to the incoherent torsional lattice vibrations. ${ }^{21}$

However, as shown in Figure 10, the vertical shitt was unnecessary for the superpositions of $G^{\prime}$ and $G^{\prime \prime}$ curves of the original samples of single crystals at temperatures below about $100^{\circ} \mathrm{C}$ and $115^{\circ} \mathrm{C}$ respectively. 


\section{K. MikI}

The temperature variation of rigidity is obviously dependent upon the fine structure of the sample. The temperature variation of rigidity for the melt-crystallized polyoxymethylene might be closely related to the lattice defects included in the crystal, as suggested by Takayanagi. ${ }^{36}$ As described previously, the amplitude of the thermal vibration of the chain molecules in the crystals increases furthermore at about $40^{\circ} \mathrm{C} .{ }^{27}$ This temperature corresponds to the temperature at which the temperature variation of rigidity starts. Thus the large amplitude lattice vibration of the crystal is also connected with the temperature variation of the rigidity as follows.

At lower temperatures the lattice distortions of the crystal are presumably localized around the lattice defects included originally in the crystal. The thermal motion of the chain molecules in the crystal increases in amplitude with increasing temperature. Consequently the lattice distortions begin to spread at about $40^{\circ} \mathrm{C}$ in order to relax the stress which is concentrated around the lattice defects. The spread of the lattice distortions due to the thermal motion of the molecules causes a "smearing-out effect" of the intermolecular potential of the crystal. If these distortions spread only along the normal direction to the $c$-axis of the crystal, the component $c_{66}$ should decrease effectively with increasing temperature. ${ }^{15}$ The temperature variation of the rigidity of the crystal does not occur in the single crystals, because the single crystals include less lattice defects than the melt-crystallized polyoxymethylene. As shown in Figure 10 , the rigidity decreases with increasing temperature above about $50^{\circ} \mathrm{C}$ for the heat-treated single crystal mat. This is presumably due to the lattice defects caused by heat-treatment of the sample.

The lattice defects which are frozen in the non-equilibrium states will be partly diminished with gamma-ray irradiation for the melt-crystallized polyoxymethylene. This problem will be discussed in another paper.

Acknowledgements. The author is grateful to Professor M. Kaneko for the interest shown in this work. Thanks are also due to Mr. S. Nakamura for his excellent technical assistance in the preparation of the sample and to Mr. A.
Nobuta and Mr. A. Chiba for their assistance in taking X-ray small angle scattering patterns.

\section{REFERENCES}

1. E. Sauter, Phys. Chem., 2, 186 B (1933).

2. H. Tadokoro, T. Yasumoto, S. Murahashi and I. Nitta, J. Polym. Sci., 44, 266 (1960).

3. N. G. McCrum, J. Polym. Sci., 54, 561 (1961).

4. B. E. Read and G. Williams, Polymer, 2, 239 (1961).

5. R. E. Wetton and G. Allen, Polymer, 7, 331 (1966).

6. Y. Ishida, Kolloid-Z., 171, 149 (1960).

7. M. Kakizaki, Y. Morita, K. Tsuge and T. Hideshima, Rep. Prog. Polym. Phys., Japan, 10, 397 (1967).

8. K. Miki, S. Yamane and M. Kaneko, Rep. Prog. Polym. Phys., Japan, 12, 277 (1969); Proceedings of the Fifth International Congress on Rheology, Vol. 3, University of Tokyo Press, 1970, p 335.

9. J. D. Ferry, J. Amer. Chem. Soc., 72, 3736 (1950).

10. M. L. Williams, R. F. Landel and J. D. Ferry, J. Amer. Chem. Soc., 77, 3701 (1955).

11. A. Keller, Philos. Mag., 2, 1171 (1957).

12. P. H. Geil, N. K. J. Symons and R. G. Scott, J. Appl. Phys., 30, 1516 (1959).

13. M. Takayanagi, S. Minami, K. Neki and A. Nagai, Zairyo (J. Soc. Material Sci. Japan), 14, 343 (1965).

14. K. Miki, K. Hikichi and M. Kaneko, Japan. J. Appl. Phys., 6, 931 (1967).

15. K. Miki, J. Yasukawa and M. Kaneko, Japan. J. Appl. Phys., 8, 159 (1969).

16. M. Takayanagi, T. Aramaki, M. Yoshino and K. Hoashi, J. Polym. Sci., 46, 531 (1960).

17. Y. Wada and K. Tsuge, Japan. J. Appl. Phys., 1, 64 (1962).

18. K. Tsuge, H. Enjoji, H. Terada, Y. Ozawa and Y. Wada, Japan. J. Appl. Phys., 1, 2701 (1962).

19. K. Okano, Riken-Hokoku (Soc. Pap. Inst. Phys. Chem. Res.) Tokyo, 40, 295 (1964).

20. A. Peterlin and E. W. Fischer, Z. Phys., 159, 272 (1960).

21. A. Peterlin, E. W. Fischer and Chr. Reinhold, J. Chem. Phys., 37, 1043 (1962).

22. K. Tsuge, Japan. J. Appl. Phys., 3, 588 (1964).

23. K. Arisawa, K. Tsuge and Y. Wada, Japan. J. Appl. Phys., 4, 138 (1965).

24. K. Miki, Y. Yokokawa, K. Hikichi and J. Furuichi, Japan. J. Appl. Phys. 5, 818 (1966).

25. K. M. Sinnott, J. Appl. Phys., 37, 3385 (1966). 
Dynamic Mechanical Properties of Polyoxymethylene

26. M. Takayanagi and T. Matsuo, J. Macromol. Sci. (Phys.), B 1 (3), 407 (1967).

27. Y. Aoki, A. Chiba and M. Kaneko, J. Phys. Soc. Japan, 27, 1579 (1969).

28. A. Nobuta, A. Chiba and M. Kaneko, Rep. Prog. Polym. Phys. Japan, 12, 137 (1969).

29. E. W. Fischer, Kolloid-Z. Z. Polym., 218, 97, (1967).

30. J. M. Schultz, W. H. Robinson and G. M. Pound, J. Polym. Sci., Part A-2, 511 (1967).

31. C. F. Hammer, T. A. Koch and J. F. Whitney, J. Appl. Polym. Sci., 1, 169 (1959).

32. S. Okamura, K. Hayashi and Y. Kitanishi, $J$.
Polym. Sci., 58, 925 (1962).

33. K. Hayashi, H. Ochi, M. Nishii, Y. Miyake and S. Okamura, J. Polym. Sci., Part B-1, 427 (1963).

34. N. Yamada, Z. Orito and S. Minami, J. Polym. Sci., Part A-3, 4173 (1965).

35. M. Takayanagi, K. Imada, A. Nagai, T. Tatsumi and T. Matsuo, J. Polym. Sci., Part C-16, 867 (1967).

36. M. Takayanagi, K. Imada and T. Kajiyama, J. Polym. Sci., Part C-15, 263 (1966).

37. E. W. Fischer, H. Goddar and C. F. Schmidt, Kolloid Z. Z. Polym., 226, 30 (1968). 